\title{
THE INNER AND OUTER SPACE OF 2-DIMENSIONAL LAGUERRE PLANES
}

\author{
B. POLSTER and G. F. STEINKE
}

(Received 4 May 1995)

Communicated by J. R. J. Groves

\begin{abstract}
The classical 2-dimensional Laguerre plane is obtained as the geometry of non-trivial plane sections of a cylinder in $\mathbb{R}^{3}$ with a circle in $\mathbb{R}^{2}$ as base. Points and lines in $\mathbb{R}^{3}$ define subsets of the circle set of this geometry via the affine non-vertical planes that contain them. Furthermore, vertical lines and planes define partitions of the circle set via the points and affine non-vertical lines, respectively, contained in them.

We investigate abstract counterparts of such sets of circles and partitions in arbitrary 2-dimensional Laguerre planes. We also prove a number of related results for generalized quadrangles associated with 2-dimensional Laguerre planes.
\end{abstract}

1991 Mathematics subject classification (Amer. Math. Soc.): 51B15, 51H15.

Keywords and phrases: Laguerre plane, topological incidence geometry, generalized quadrangle.

\section{Introduction}

Let us first recall the definition of 2-dimensional Laguerre planes. Starting with a very general definition of these incidence structures it has been shown that all of them can be represented in a certain normal form. We incorporate this normal form in our definition; cf. [Gr1, Gr2].

A 2-dimensional (or flat) Laguerre plane $L=\left(\mathbb{S}^{1} \times \mathbb{R}, \mathscr{C}, \|\right)$ is an incidence structure consisting of a point set, a circle set and an equivalence relation (parallelism) defined on the point set. Incidence is defined by inclusion. The point set is the cylinder $\mathbb{S}^{1} \times \mathbb{R}$, the circles are graphs of continuous functions $\mathbb{S}^{1} \rightarrow \mathbb{R}$, and the equivalence classes (parallel classes) of $\|$ are the verticals in $\mathbb{S}^{1} \times \mathbb{R}$, that is, the sets $\{(a, y) \mid y \in \mathbb{R}\}, a \in \mathbb{S}^{1}$. Furthermore, the incidence structure has to satisfy the

This research was supported by a Feodor Lynen fellowship.

(C) 1997 Australian Mathematical Society 0263-6115/97 \$A2.00+0.00 
following axioms:

(L1) Three pairwise non-parallel points are contained in a uniquely determined circle.

(L2) For two non-parallel points $p, q$ and a circle $c$ through $p$ there exists a uniquely determined circle through $q$ that touches $c$ at $p$, that is, intersects $c$ only in the point $p$, or coincides with $c$.

The classical 2-dimensional Laguerre plane is obtained as the geometry of nontrivial plane sections of a cylinder in $\mathbb{R}^{3}$ with a circle in $\mathbb{R}^{2}$ as base, or equivalently, as the geometry of non-trivial plane sections of an elliptic cone, in real 3-dimensional projective space, with its vertex removed.

The set of circles in this geometry that corresponds to the set of planes through some point in $\mathbb{R}^{3}$ takes on three different forms depending on whether the point is inside the cylinder, on the cylinder, or outside the cylinder. These sets have counterparts in non-classical 2-dimensional Laguerre planes and 'separate' the circle sets of these Laguerre planes into two open components. If two 2-dimensional Laguerre planes share the same separating set, a new 2-dimensional Laguerre plane can be constructed by combining the circles of the separating set and the circles of two open components of the separating set, taken from the two planes, into the circle set of a new 2dimensional Laguerre plane. Separating sets like this have already been investigated in [PS2].

Every vertical line in $\mathbb{R}^{3}$ gives rise to a special kind of partition of the circle set of the classical 2-dimensional Laguerre plane. The elements of the partition are the sets of circles that correspond to the points in the line. Counterparts of such partitions in non-classical 2-dimensional Laguerre planes also exist.

The set of circles that corresponds to a non-vertical line in $\mathbb{R}^{3}$ corresponds to a pencil of circles through two points, to a pencil of circles that touch in a point, and to a flock of circles in the classical 2-dimensional Laguerre plane, depending on whether the line is a secant, a tangent or an exterior line of the cylinder.

Every vertical plane in $\mathbb{R}^{3}$ gives rise to a special kind of partition of the circle set of the classical 2-dimensional Laguerre plane. The elements of the partition are the sets of circles that correspond to the non-vertical lines in the plane. The most interesting such partitions arise from planes that do not intersect the cylinder. These are partitions of the circle set into flocks. Such a partition is called a resolution. We construct examples of resolutions in non-classical Laguerre planes.

The partitions of the circle set associated with non-vertical lines and non-vertical planes are quite messy and will not really be investigated in this paper.

In a final section we collect related results for the generalized quadrangles associated with 2-dimensional Laguerre planes. We construct separating sets in such quadrangles from separating sets in Laguerre planes. 
We take the opportunity to collect some more facts and definitions that we will need in the following.

The common point set of 2-dimensional Laguerre planes $\mathbb{S}^{1} \times \mathbb{R}$ is a metrizable 2dimensional topological space. Circles are homeomorphic to the unit circle $\mathbb{S}^{1}$. When the circle sets are topologized by the Hausdorff metric with respect to a metric that induces the topology of the point set, then the planes are topological in the sense that the operations of joining three points by a circle, intersecting of circles, and touching are continuous with respect to the induced topologies on their respective domains of definition.

A 2-dimensional projective plane $\mathscr{P}=(P, \mathscr{L})$ is a projective plane whose point set $P$ is the real projective plane (viewed as a 2 -dimensional topological space) and whose lines are subsets of $P$ homeomorphic to the unit circle $\mathbb{S}^{1}$. The affine plane one arrives at by deleting a line from $\mathscr{P}$ has a point set that is homeomorphic to $\mathbb{R}^{2}$. Its lines all separate the point set in two open components and are homeomorphic to $\mathbb{R}$. Every affine plane of this form is called a 2-dimensional affine plane and it can be shown that the projective extension of such a plane can be turned into a 2-dimensional projective plane in a unique way. The classical examples of 2-dimensional affine and projective planes are the affine and projective planes over the real numbers.

Let $\mathscr{S}=\left(\mathbb{S}^{1} \times \mathbb{R}, \mathscr{C}, \|\right)$ be an incidence structure with a point set $\mathbb{S}^{1} \times \mathbb{R}$ and circle set $\mathscr{C}$ and let the parallelism on the point set be defined as in the special case of a 2-dimensional Laguerre plane. Let $p$ be a point in $\mathbb{S}^{1} \times \mathbb{R}$. Then $\mathscr{C}_{p}$ denotes the set of circles in $\mathscr{S}$ passing through $p$. Associated with every point $p \in \mathbb{S}^{1} \times \mathbb{R}$ is a derived incidence structure $\mathscr{S}_{p}$ whose point set consists of all points not parallel to $p$ and whose line set consists of all circles in $\mathscr{C}_{p}$ that have been punctured at $p$ and all parallel classes that do not contain $p$. It is well-known that the incidence structure $\mathscr{S}$ is a 2-dimensional Laguerre plane if and only if the derived incidence structures at all its points are 2-dimensional affine planes (see, for example, [PS1]).

\section{Ovoidal planes and the non-classical Laguerre planes constructed by Mäurer, Hartmann and Kleinewillinghöfer}

In this section we recall some constructions of non-classical 2-dimensional Laguerre planes that are modifications of the construction of the classical Laguerre plane in $\mathbb{R}^{3}$.

We reiterate: The classical 2-dimensional Laguerre plane is obtained as the geometry of non-trivial plane sections of a cylinder in $\mathbb{R}^{3}$ with a circle in the $x y$-plane as base.

If we replace the circle in this construction by an arbitrary topological oval in the $x y$-plane, that is, a differentiable, convex simply closed curve, we get one of the so-called ovoidal Laguerre planes. These Laguerre planes are not isomorphic to the 
classical Laguerre plane if the oval under consideration is not an ellipse.

Mäurer constructed non-classical Laguerre planes by replacing the non-vertical planes that intersect the cylinder by certain bent planes [Mäu]. We describe his construction in a way that will prove very useful in the following sections.

Let the circle in the classical construction be a circle in the $x y$-plane with center at the origin. Let $A$ be an arbitrary vertical plane parallel to the $x z$-plane. Then every non-vertical plane $E$ in $\mathbb{R}^{3}$ intersects $A$ and the $y z$-plane in one line each. Let $a$ be the first line and let $p$ be the infinite point of the second line. Then $E$ can be considered as the set of all points in $\mathbb{R}^{3}$ that get projected on $a$ through $p$.

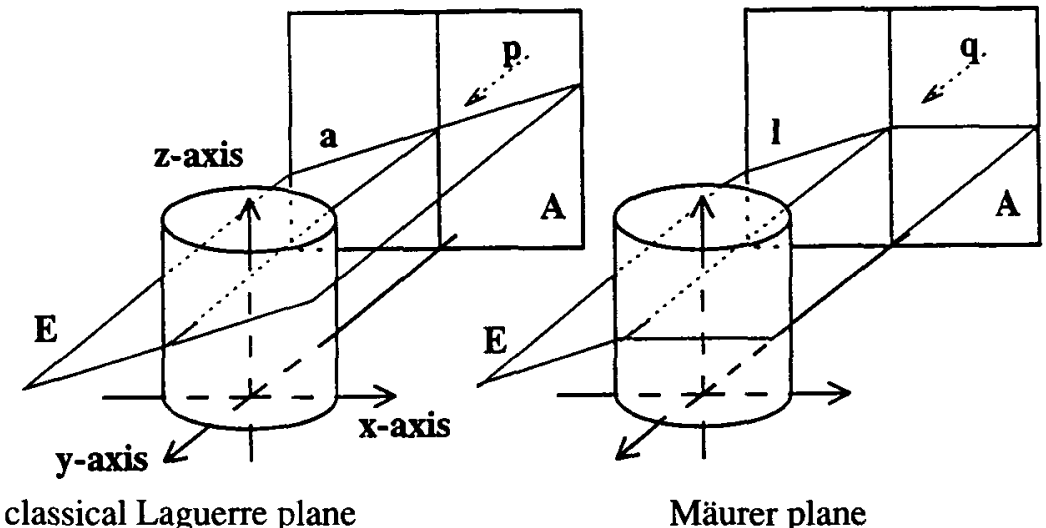

FIGURE 1

The plane $A$ considered as an incidence structure with a set of points and a set of lines is, of course, just a copy of the real affine plane. We now replace the non-vertical lines in $A$ by lines that are bent at the $y z$-plane, such that the resulting incidence structure is a Moulton plane (cf. [Mou, St1]), that is, a very special kind of 2-dimensional affine plane. Let $l$ be a (bent) line in this plane and let $q$ be an infinite point of one of the lines in the $y z$-plane. Then the set of all points in $\mathbb{R}^{3}$ that gets projected from $q$ onto $l$ is a 'bent' plane (see Figure 1). Let $B$ be the set of all such bent planes. Now, the geometry of sections of these bent planes with the cylinder is (in general) a non-classical Laguerre plane. Since all translations in the vertical direction are collineations of Moulton planes, all parallel translates of $A$ (as a Moulton plane) in directions given by vectors in the $y z$-plane will give exactly the same Laguerre plane.

This construction has been generalized further by Kleinewillinghöfer [Kl] who showed that we still get 2-dimensional Laguerre planes if we replace the circle in Mäurer's constructions by ovals that intersect the $y z$-plane at right angles. Some of the planes in [St3] are also generalizations of Mäurer's planes. Here the plane $A$ is replaced by a generalized Moulton plane [St1] in which the lines are not only bent at the $y z$-plane, but also broken (that is, they are no longer continuous curves). We can 
also use the generalized Moulton planes together with ovals that intersect the $y z$-plane at right angles to arrive at a class of 2-dimensional Laguerre planes that includes all Laguerre planes mentioned before (see [PRS]). In the following we will refer to the Laguerre planes that arise from Mäurer's construction as Mäurer planes.

Finally, we mention that Hartmann [Ha] also generalized Mäurer's construction. His variation yields Laguerre planes that are no longer topological.

\section{Points-separating sets in 2-dimensional Laguerre planes}

Let us have a look at the different sets of circles in the classical, or more generally, in an ovoidal, 2-dimensional Laguerre plane that correspond to the sets of planes through points in $\mathbb{R}^{3}$. If $p \in \mathbb{R}^{3}$, let $C_{p}$ be the associated set of circles.

If $p$ is contained in the cylinder, then $C_{p}$ is, essentially, the set of lines in the derived affine plane at the point $p$.

Let $p$ be contained in the interior of the cylinder and let $S$ be a sphere with center at $p$ that is completely contained in the interior of the cylinder (Figure 2). Let $q_{1}$ and $q_{2}$ be the points of intersection of the vertical line through $p$ with $S$. Then $S \backslash\left\{q_{1}, q_{2}\right\}$ and the cylinder are in 1-1 correspondence via the projection with center $p$. Under this projection the circles in $C_{p}$ correspond to the great circles on $S$ that do not pass through $q_{1}$ and $q_{2}$ (pairs of parallel classes correspond to these circles). So, essentially, $C_{p}$ is the geometry of great circles on $S$, which is just the 'double cover' of the real projective plane. Associated with $p$ is an involutory homeomorphism $\gamma_{p}$ of the cylinder to itself that maps a point on the cylinder to the second point of intersection of the line through the point and $p$ with the cylinder. This involution is fixed-point-free and orientation-reversing. Furthermore, its fixed circle set is $C_{p}$. If $p$ is on the $z$-axis and the Laguerre plane is the classical one in its usual representation, then the involution is an automorphism of the Laguerre plane and there exist circles $c$ such that $c \cap \gamma_{p}(c)=\emptyset$. This automorphism is induced by a central collineation of $\mathbb{R}^{3}$ which is a reflection with centre $p$.

If $p$ is situated outside the cylinder, let $V$ be a vertical plane in $\mathbb{R}^{3}$ such that the cylinder and the point $p$ lie in different connected components of $\mathbb{R}^{3} \backslash V$ (see Figure 3 ), and let $V_{p}$ be the set of points in $V$ that are hit by lines in $\mathbb{R}^{3}$ that contain $p$ and some point on the cylinder. The set $V_{p}$ is a vertical strip in an $\mathbb{R}^{2}$ over a closed interval. The non-vertical lines in $V$ induce a line geometry on this strip and it is again easy to see, via projection through $p$, that $C_{p}$ is essentially a 'double cover' of this incidence geometry (actually it is a double cover only of the open interior of the strip $V_{p}$.) We can also associate with $p$, in the same manner as before, an involutory homeomorphism $\gamma_{p}$ of the cylinder to itself. This involution fixes two parallel classes $\Pi_{0}, \Pi_{\infty}$ pointwise. Again, in the classical case, this involution is an automorphism 


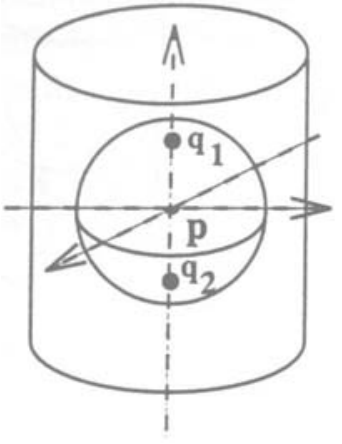

FIGURE 2

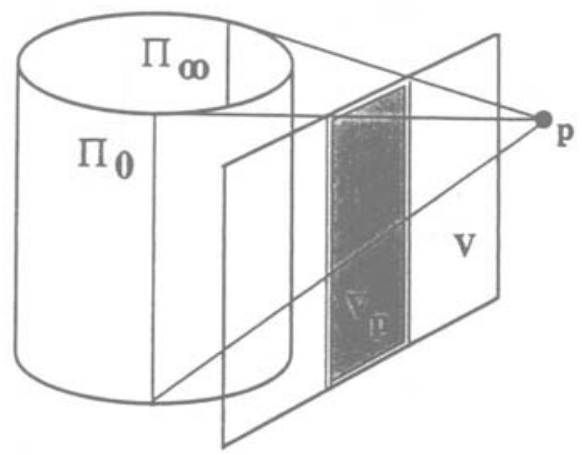

FIGURE 3

of the Laguerre plane. This automorphism is induced by a central collineation of real projective 3-space which is a reflection at the plane through $\Pi_{0}$ and $\Pi_{\infty}$ with centre $p$.

It is clear that if we replace the cylinder over the circle and the cutting planes by cylinders over ovals and bent planes, respectively, as described in Section 2, we get sets $C_{p}$ in the Mäurer planes. Let $p$ be a point on the $y z$-plane. As in the classical model we find that if $p$ is contained in the cylinder, or outside the cylinder, then $C_{p}$ is, essentially, the derived affine plane in $p$, or a double cover of a strip taken from a 2-dimensional affine plane. Actually, the affine plane we just mentioned is, essentially, the Moulton plane in the Mäurer construction. If $p$ is situated outside the cylinder, we can also associate with it, as in the classical case, an involutory homeomorphism of the cylinder to itself that has $C_{p}$ as its fixed circle set.

Separating sets. Let $L=\left(\mathbb{S}^{1} \times \mathbb{R}, \mathscr{C}, \|\right)$ be a 2-dimensional Laguerre plane. For each $p \in \mathbb{S}^{1} \times \mathbb{R}$ let $\mathscr{C}_{p}$ be the set of all circles that pass through $p$ and let $\mathscr{C}_{p^{+}}$and $\mathscr{C}_{p^{-}}$be the collection of all circles that intersect the parallel class of $p$ above or below $p$, respectively.

In [PS2, Proposition 5.1] we proved the following

PROPOSITION 1. Let $L=\left(\mathbb{S}^{1} \times \mathbb{R}, \mathscr{C}, \|\right)$ and $L^{*}=\left(\mathbb{S}^{1} \times \mathbb{R}, \mathscr{C}^{*}, \|\right)$ be two 2dimensional Laguerre planes. Let $p \in \mathbb{S}^{1} \times \mathbb{R}$ and suppose $\mathscr{C}_{p}=\mathscr{C}_{p}^{*}$. Then $L \mid L^{*}=\left(\mathbb{S}^{1} \times \mathbb{R}, \mathscr{C}_{p} \cup \mathscr{C}_{p^{+}} \cup \mathscr{C}_{p^{-}}, \|\right)$is a 2-dimensional Laguerre plane (Figure 4).

Similarly, let $\gamma$ be an involutory automorphism of $L$, and let $\mathscr{C}_{\gamma}$ be its fixed circle set. We note that every automorphism of a 2-dimensional Laguerre plane is a homeomorphism of its point set to itself. If $\gamma$ is fixed-point-free and there exists a circle such that $c \cap \gamma(c)=\emptyset$, we call $\gamma$ an inversion of $L$. If $\gamma$ fixes two parallel classes pointwise, we call it a reflection of $L$. It is clear where these names come from: In the classical model over the unit circle, the reflection through one of the points on 


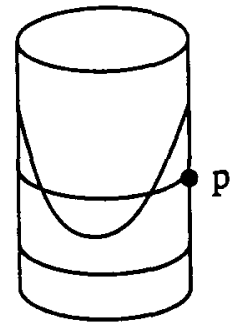

$L$

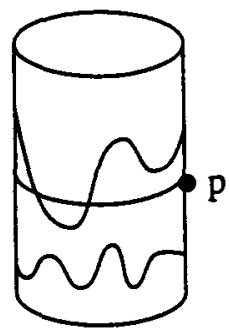

$L^{*}$

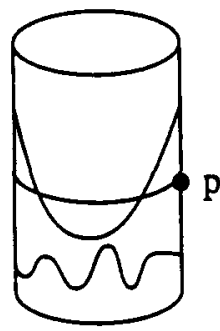

$L \mid L^{*}$

FIGURE 4

the $z$-axis induces an inversion of the classical Laguerre plane. The reflection of the cylinder through one of the outer points of the cylinder induces a reflection of the Laguerre plane.

Let $\gamma$ be an inversion of $L$. Then every circle that is not fixed by $\gamma$ is disjoint from its image under $\gamma[\mathrm{Gr} 3,3.4]$. Let $\mathscr{C}_{\gamma^{+}}\left(\mathscr{C}_{\gamma^{-}}\right)$be the set of all circles $c \in \mathscr{C}$ such that on the cylinder $c$ lies above (below) $\gamma(c)$. In [PS2, Proposition 5.2] we proved

Proposition 2. Let $L=\left(\mathbb{S}^{1} \times \mathbb{R}, \mathscr{C}, \|\right)$ and $L^{*}=\left(\mathbb{S}^{1} \times \mathbb{R}, \mathscr{C}^{*}, \|\right)$ be two 2dimensional Laguerre planes. Suppose both Laguerre planes admit the inversion $\gamma$ and that $\mathscr{C}_{\gamma}=\mathscr{C}_{\gamma}^{*}$. Then $L \mid L^{*}=\left(\mathbb{S}^{1} \times \mathbb{R}, \mathscr{C}_{\gamma} \cup \mathscr{C}_{\gamma^{+}} \cup \mathscr{C}_{\gamma^{-}}^{*}, \|\right)$ is a 2-dimensional Laguerre plane (Figure 5).

c

$\gamma(\mathbf{c})$

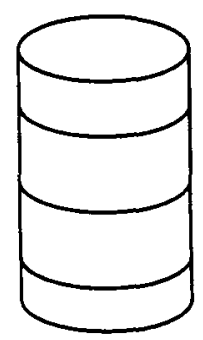

$L$

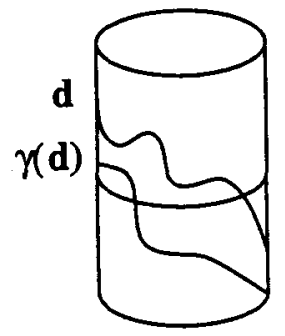

$L^{*}$

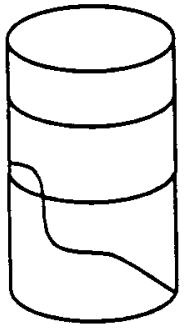

$L \mid L^{*}$

FIGURE 5

These two results show that it makes sense to think of the sets $\mathscr{C}_{p}$ and $\mathscr{C}_{\gamma}$ as separating sets. Both sets separate the circle set $\mathscr{C}$ in yet another sense. The circle set of a 2-dimensional Laguerre plane is homeomorphic to $\mathbb{R}^{3}$. We already noted in [PS2] that both $\mathscr{C}_{p}$ and $\mathscr{C}_{\gamma}$ are closed subsets of the circle set homeomorphic to $\mathbb{R}^{2}$. In particular, both sets separate the circle set into two open components. 
Our considerations above suggest looking for a third kind of separating sets that arises from reflections. Let $\gamma$ be a reflection of $L$. Let $\Pi_{0}$ and $\Pi_{\infty}$ denote the two parallel classes that are pointwise fixed by $\gamma$ and let $H_{1}$ and $H_{2}$ be the two connected components of $\left(\mathbb{S}^{1} \times \mathbb{R}\right) \backslash\left(\Pi_{0} \cup \Pi_{\infty}\right)$. Clearly, if $c \in \mathscr{C}$ is a circle that is not fixed by $\gamma$, then $c$ and $\gamma(c)$ intersect in exactly two points, one in $\Pi_{0}$, the other one in $\Pi_{\infty}$, that is, $c \cap \gamma(c)=c \cap\left(\Pi_{0} \cup \Pi_{\infty}\right)$. Let $\mathscr{C}_{\gamma^{+}}\left(\mathscr{C}_{\gamma^{-}}\right)$be the set of all circles $c \in \mathscr{C}$ such that $c$ lies above (below) $\gamma(c)$ on $H_{1}$.

Proposition 3. Let $L=\left(\mathbb{S}^{1} \times \mathbb{R}, \mathscr{C}, \|\right)$ and $L^{*}=\left(\mathbb{S}^{1} \times \mathbb{R}, \mathscr{C}^{*}, \|\right)$ be two 2dimensional Laguerre planes. Suppose both Laguerre planes admit the reflection $\gamma$ and that $\mathscr{C}_{\gamma}=\mathscr{C}_{\gamma}^{*}$. Then $L \mid L^{*}=\left(\mathbb{S}^{1} \times \mathbb{R}, \mathscr{C}_{\gamma} \cup \mathscr{C}_{\gamma^{+}} \cup \mathscr{C}_{\gamma^{-}}^{*}, \|\right)$ is a 2-dimensional Laguerre plane (Figure 6).

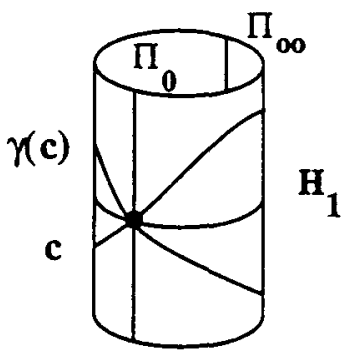

$L$

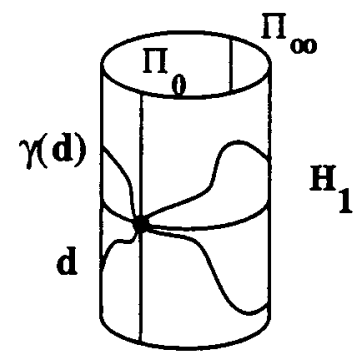

$L^{*}$

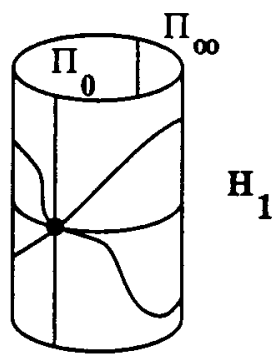

$L \mid L^{*}$

FIGURE 6

Propositions 2 and 3 are corollaries of Propositions $2^{*}$ and $3^{*}$, respectively, that we are going to prove a little further down in the text.

Groh proved that all 2-dimensional Laguerre planes that admit inversions arise from 2-dimensional Möbius planes via the construction described in [Gr3] and [Gr4]. The discussion in [Sch3] yields that all 2-dimensional Laguerre planes that admit reflections arise from 2-dimensional Minkowski planes. We will say some more about these characterization of 2-dimensional Laguerre planes admitting inversions and reflection in Section 5, Remark 3.

Propositions 2 and 3 do not really say anything about most of the sets $C_{p}$, and their associated involutions of the cylinder, that we considered at the beginning of this section (most of these involutions are not automorphisms of the respective Laguerre planes). It turns out that both propositions can be generalized to accomodate these less specialized sets and involutions.

Let $L=\left(\mathbb{S}^{1} \times \mathbb{R}, \mathscr{C}, \|\right)$ be a 2-dimensional Laguerre plane, and let $\gamma$ be an involutory homeomorphism of $\mathbb{S}^{1} \times \mathbb{R}$ to itself that is not the identity and that has the following properties: 
(1) it maps parallel classes to parallel classes; and

(2) for all $p \in \mathbb{S}^{1} \times \mathbb{R}$ for which $p \neq \gamma(p)$ every circle through $p$ and $\gamma(p)$ is (globally) fixed by $\gamma$.

We call $\gamma$ a pre-inversion of $L$ if $\gamma$ is fixed-point-free and orientation-reversing. We call it pre-reflection if its fixed point set consists of two distinct parallel classes $\Pi_{0}$ and $\Pi_{\infty}$. As in the case of reflections let $H_{1}$ and $H_{2}$ denote the two connected components of $\left(\mathbb{S}^{1} \times \mathbb{R}\right) \backslash\left(\Pi_{0} \cup \Pi_{\infty}\right)$.

LEMMA 2. Let $L=\left(\mathbb{S}^{1} \times \mathbb{R}, \mathscr{C}, \|\right)$ be a 2-dimensional Laguerre plane and let $\gamma$ be an involutory homeomorphism of $\mathbb{S}^{1} \times \mathbb{R}$ to itself.

(1) If $\gamma$ is an inversion, or a reflection of $L$, then it is a pre-inversion, or pre-reflection, respectively, of $L$.

(2) If $\gamma$ is a pre-inversion of $L$ and $c$ is a circle that is not fixed by $\gamma$, then $c \cap \gamma(c)=\emptyset$.

(3) If $\gamma$ is a pre-reflection of $L$ and $c$ is a circle that is not fixed by $\gamma$, then $c \cap \gamma(c)=$ $c \cap\left(\Pi_{0} \cup \Pi_{\infty}\right)$.

PROOF. (1) By [Gr4, 3.1, 3.3], we know that every inversion is a pre-inversion. For the second part let $p \in H_{1} \cup H_{2}$, let $c$ be a circle through $p$ and $\gamma(p)$ and let $q=c \cap \Pi_{0}$. Then $\gamma(c)$ contains the points $\gamma(p), \gamma(\gamma(p))=p$ and $\gamma(q)=q$. Hence $c=\gamma(c)$.

(2) Let $\gamma$ be a pre-inversion. Assume $c$ is a circle that is not fixed by $\gamma$. If there exists a $p \in c \cap \gamma(c)$, then $\gamma(p)$ is also contained in $c \cap \gamma(c)$. By assumption, $p \neq \gamma(p)$. Hence $c$ is fixed by $\gamma$. This is a contradiction.

(3) Let $\gamma$ be a pre-reflection. Let $c$ be a circle that is not fixed by $\gamma$. Then it intersects its image under $\gamma$ in two points; one on $\Pi_{0}$, a second one on $\Pi_{\infty}$. As above, the assumption that there exists one further point that is contained in both $c$ and $\gamma(c)$ leads to a contradiction.

With this lemma at hand, it makes sense to define the sets $\mathscr{C}_{\gamma}, \mathscr{C}_{\gamma^{+}}$, and $\mathscr{C}_{\gamma^{-}}$as in the case of reflections and inversions. We can generalize Propositions 2 and 3 as follows:

Proposition 2*. Let $L=\left(\mathbb{S}^{1} \times \mathbb{R}, \mathscr{C}, \|\right)$ and $L^{*}=\left(\mathbb{S}^{1} \times \mathbb{R}, \mathscr{C}^{*}, \|\right)$ be two 2dimensional Laguerre planes. Suppose both Laguerre planes admit the pre-inversion $\gamma$ and that $\mathscr{C}_{\gamma}=\mathscr{C}_{\gamma}^{*}$. Then $L \mid L^{*}=\left(\mathbb{S}^{1} \times \mathbb{R}, \mathscr{C}_{\gamma} \cup \mathscr{C}_{\gamma^{+}} \cup \mathscr{C}_{\gamma-}^{*},||\right)$ is a 2-dimensional Laguerre plane.

PROPOSITION $3^{*}$. Let $L=\left(\mathbb{S}^{1} \times \mathbb{R}, \mathscr{C}, \|\right)$ and $L^{*}=\left(\mathbb{S}^{1} \times \mathbb{R}, \mathscr{C}^{*}, \|\right)$ be two 2dimensional Laguerre planes. Suppose both Laguerre planes admit the pre-reflection $\gamma$ and that $\mathscr{C}_{\gamma}=\mathscr{C}_{\gamma}^{*}$. Then $L \mid L^{*}=\left(\mathbb{S}^{1} \times \mathbb{R}, \mathscr{C}_{\gamma} \cup \mathscr{C}_{\gamma^{+}} \cup \mathscr{C}_{\gamma^{*}}, \|\right)$ is a 2-dimensional Laguerre plane. 
Notice that by Lemma 2(1) these results are really generalizations of Propositions 2 and 3.

To be able to prove these propositions let us recall one further result in [PS2, Proposition 1]: Let $\mathscr{P}=(P, \mathscr{L})$ be a 2-dimensional projective plane. Given two distinct points $q$ and $q^{\prime}$ on a line $l$, the complement $l \backslash\left\{q, q^{\prime}\right\}$ consists of two connected components $l^{+}$and $l^{-}$. Now we can partition $\mathscr{L}$ by $\left(\mathscr{L}_{q} \cup \mathscr{L}_{q^{\prime}}\right) \cup \mathscr{L}^{+} \cup \mathscr{L}^{-}$where $\mathscr{L}^{+}$and $\mathscr{L}^{-}$denote the collections of all lines that intersect the line $l$ through $q$ and $q^{\prime}$ in $l^{+}$and $l^{-}$, respectively, and $\mathscr{L}_{q}\left(\mathscr{L}_{q^{\prime}}\right)$ denotes the pencil of lines through the point $q\left(q^{\prime}\right)$

LEMMA 3. Let $\mathscr{P}=(P, \mathscr{L})$ and $\mathscr{P}^{*}=\left(P, \mathscr{L}^{*}\right)$ be two 2-dimensional projective planes, and suppose the line $l$ is contained in both line sets. Suppose further that $\mathscr{L} \cap \mathscr{L}^{*}$ contains the line pencils through the two distinct points $q, q^{\prime} \in l$. Then $\mathscr{P} \mid \mathscr{P}^{*}=\left(P, \mathscr{L}_{q} \cup \mathscr{L}_{q^{\prime}} \cup \mathscr{L}^{+} \cup \mathscr{L}^{*-}\right)$ is a 2-dimensional projective plane.

In order to apply this result we need one more lemma.

LEMMA 4. Let $L=\left(\mathbb{S}^{1} \times \mathbb{R}, \mathscr{C}, \|\right)$ be a 2-dimensional Laguerre plane. Let $\gamma$ be a pre-inversion or pre-reflection of $L$.

(1) Let $p$ and $q$ be points such that $q \neq \gamma(q)$ and such that $p$ is neither parallel to $q$ nor to $\gamma(q)$. Then there exists a unique circle in $\mathscr{C}_{\gamma}$ that contains both $p$ and $q$.

Let $\gamma$ be a pre-inversion of $L$.

(2) If $c^{*}$ is a circle that is fixed by $\gamma$, then $\gamma$ exchanges the two connected components of $\left(\mathbb{S}^{1} \times \mathbb{R}\right) \backslash c^{*}$.

(3) Let $p$ be a point and let $c$ be a circle that contains $p$. Then $c$ is contained in $\mathscr{C}_{\gamma}$, $\mathscr{C}_{\gamma^{+}}$, or $\mathscr{C}_{\gamma^{-}}$if and only if $c$ intersects the parallel class of $\gamma(p)$ in, above, or below $\gamma(p)$, respectively.

Let $\gamma$ be a pre-reflection of $L$.

(4) The two halves $H_{1}$ and $H_{2}$ get exchanged by $\gamma$.

(5) If $c^{*}$ is a circle that is fixed by $\gamma$, then $\gamma$ fixes the two connected components of $\left(\mathbb{S}^{1} \times \mathbb{R}\right) \backslash c^{*}($ globally $)$.

(6) Let $p$ be a point that is not fixed by $\gamma$, that is, a point that is not contained in $\Pi_{0} \cup \Pi_{\infty}$, and let $c$ be a circle that contains $p$. Then $c$ is contained in $\mathscr{C}_{\gamma}, \mathscr{C}_{\gamma^{+}}$, or $\mathscr{C}_{\gamma^{-}}$if and only if $p$ is contained in $\mathrm{H}_{2}$ and $c$ intersects the parallel class of $\gamma(p)$ in, above, or below $\gamma(p)$, respectively, or if $p$ is contained in $H_{1}$ and $c$ intersects the parallel class of $\gamma(p)$ in, below, or above $\gamma(p)$.

(7) Let $p$ be a point that is fixed by $\gamma$. Then the set of circles through $p$ that are contained in $\mathscr{C}_{\gamma}$ is a tangent pencil of circles through $p$. Let $c^{*}$ be a circle in this particular tangent pencil and let $c$ be a circle that intersects the two fixed parallel classes in the same points as $c^{*}$. Then $c$ is contained in $\mathscr{C}_{\gamma}, \mathscr{C}_{\gamma^{+}}$, or $\mathscr{C}_{\gamma^{-}}$ 
if and only if $c=c^{*}, c$ is situated above $c^{*}$ in $H_{1}$, or $c$ is situated below $c^{*}$ in $H_{1}$, respectively. Circles contained in a tangent pencil through $p$ are all contained in the same one of the three sets $\mathscr{C}_{\gamma}, \mathscr{C}_{\gamma^{+}}$, or $\mathscr{C}_{\gamma^{-}}$.

PROOF. (1) By assumption all circles through $q$ and $\gamma(q)$ are contained in $\mathscr{C}_{\gamma}$. Since $p$ is neither parallel to $q$ nor parallel to $\gamma(q)$ it is contained in precisely one such circle.

(2) Let $p$ be contained in $c^{*}$ and let $c$ be a circle through $p$ and $\gamma(p)$ distinct from $c^{*}$. By assumption $c$ is also fixed by $\gamma$. When restricted to $c, \gamma$ is a fixed-point-free involutory homeomorphism. As such it exchanges the two connected components of $c \backslash\{p, \gamma(p)\}$ (cf. [Val, 3.1]). These two connected components are contained in the two connected components of $\left(\mathbb{S}^{1} \times \mathbb{R}\right) \backslash c^{*}$. Hence the two connected components of $\left(\mathbb{S}^{1} \times \mathbb{R}\right) \backslash c^{*}$ are exchanged by $\gamma$.

(3) Let $\mathscr{C}_{p, c}$ denote the set of circles in $\mathscr{C}$ that touch $c$ at $p$. Exactly one circle $c^{*} \in C_{p, c}$ contains the point $\gamma(p)$. Let $q$ be the intersection of $c$ with the parallel class of $\gamma(p)$. If $q$ is contained in $c^{*}$, that is, $q=\gamma(p)$, then $c=c^{*}$ and $c$ is contained in $\mathscr{C}_{\gamma}$. Let $q$ be situated above (below) $\gamma(p)$. Then, by (2), $\gamma(q)$ is situated below (above) $p$. Since $c$ and $\gamma(c)$ are disjoint, by Lemma 2(2), we conclude that $c$ is situated above (below) $\gamma(c)$. Hence $c$ is contained in $\mathscr{C}_{\gamma^{+}}\left(\mathscr{C}_{\gamma^{-}}\right)$.

(4) Let $c \in C_{\gamma}$. Then the restriction of $\gamma$ to $c$ fixes exactly the two points of intersection of $c$ with $\Pi_{0}$ and $\Pi_{\infty}$. Since it does not fix any other points it exchanges the two connected components of these two points. Hence $\gamma$ exchanges $H_{1}$ and $H_{2}$.

(5) This follows from the fact that two parallel classes are pointwise fixed.

(6) Exactly one circle $c^{*} \in C_{p, c}$ contains the point $\gamma(p)$. Without loss of generality we may assume that $p$ is contained in $H_{2}$. Let $q$ be the intersection of $c$ with the parallel class of $\gamma(p)$. If $q$ is contained in $c^{*}$, then $c=c^{*}$ and $c$ is contained in $\mathscr{C}_{\gamma}$. Let $q$ be situated above (below) $\gamma(p)$. Then, by (4) and (5) $\gamma(q)$ is situated above (below) $p$ (in $H_{1}$ ). Lemma 2(3) yields that $c$ is situated above (below) $\gamma(c)$ in $H_{1}$. Hence $c$ is contained in $\mathscr{C}_{\gamma^{+}}\left(\mathscr{C}_{\gamma^{-}}\right)$.

(7) As a consequence of (1), we know that there exist circles in $\mathscr{C}_{\gamma}$ that contain $p$. Let $d^{*}$ be such a circle and let $d$ be any other circle that contains $p$. As a consequence of (4) and (5) we only have to see how $d$ and $d^{*}$ intersect in a neighborhood of $p$ to decide to which of the three sets of circles under discussion $d$ belongs. If $d$ touches $d^{*}$ at $p$, then $d$ is contained in $\mathscr{C}_{\gamma}$. If $d$ is situated above (below) $d^{*}$ in $H_{1}$ in a neighborhood of $p$, then $d$ belongs to $\mathscr{C}_{\gamma^{+}}\left(\mathscr{C}_{\gamma^{-}}\right)$. Now the rest of the claim follows immediately.

PROOF OF PROPOSITIONS 2* AND 3*. Let $\overline{\mathscr{C}}=\mathscr{C}_{\gamma} \cup \mathscr{C}_{\gamma^{+}} \cup \mathscr{C}_{\gamma^{-}}^{*}$. We prove that the incidence structure $\mathscr{S}=\left(\mathbb{S}^{1} \times \mathbb{R}, \overline{\mathscr{C}}, \|\right)$ is a 2-dimensional Laguerre plane. We show this by verifying for all points $p \in \mathbb{S}^{1} \times \mathbb{R}$ that the derived incidence structure $\mathscr{S}_{p}$ 
is a 2-dimensional affine plane. Let $\bar{L}_{p}$ and $\bar{L}_{p}^{*}$ denote the projective completions of the derived 2-dimensional affine planes $L_{p}$ (these projective completions are 2dimensional projective planes).

Let $p$ be one of the points that is not fixed by $\gamma$. Then Lemma 4(3) and Lemma 4(6) guarantee that Lemma 3 can be applied to the two 2-dimensional projective planes $\bar{L}_{p}$ and $\bar{L}_{p}^{*}$ where $q$ (as in Lemma 3 ) is the ideal point of the verticals in both $L_{p}$ and $L_{p}^{*}$ and $q^{\prime}$ (as in Lemma 3 ) is $\gamma(p)$. The incidence structure $\mathscr{S}_{p}$ that we are interested in is just the 2-dimensional affine plane that we arrive at by removing the uniquely determined line through $q$, that does not correspond to a vertical, from $\bar{L}_{p} \mid \bar{L}_{p}^{*}$.

Let $p$ be one of the points that is fixed by $\gamma$. This case only occurs if $\gamma$ is a pre-reflection. Note that a tangent pencil of circles through $p$ is a parallel class of lines in the affine planes $L_{p}$ and $L_{p}^{*}$. Then Lemma 4(7) guarantees that Lemma 3 can be applied to the two 2-dimensional projective planes $\bar{L}_{p}$ and $\bar{L}_{p}^{*}$ where $q$ is the ideal point of the verticals in both $L_{p}$ and $L_{p}^{*}$ and $q^{\prime}$ is the ideal point of those (common) lines in $L_{p}$ and $L_{p}^{*}$ that come from elements of $\mathscr{C}_{\nu}$. The incidence structure $\mathscr{S}_{p}$ that we are interested in is the 2-dimensional affine plane that we arrive at by removing the uniquely determined line through $q$ and $q^{\prime}$ from $\bar{L}_{p} \mid \bar{L}_{p}^{*}$.

In [PS2, Proposition 6] we found one further kind of separating set. We recall our result for the sake of completeness.

Let $L=\left(\mathbb{S}^{1} \times \mathbb{R}, \mathscr{C}, \|\right)$ be a 2 -dimensional Laguerre plane and let $c_{0} \in \mathscr{C}$. Consider the collection $\mathscr{C}^{1}$ of all circles that touch $c_{0}$. The circle $c_{0}$ separates $\mathbb{S}^{1} \times \mathbb{R}$ into two connected components $C^{+}$and $C^{-}$. We define $\mathscr{C}^{ \pm}$to be the collection of all circles that are completely contained in $C^{ \pm}$. Finally, let $\mathscr{C}^{2}$ be the set of all circles that intersect $c_{0}$ in precisely two points. Clearly, $\mathscr{C}^{1} \cup \mathscr{C}^{2} \cup \mathscr{C}^{+} \cup \mathscr{C}^{-}$is a partition of the circle set.

PROPOSITION 4. Let $L_{i}=\left(\mathbb{S}^{1} \times \mathbb{R}, \mathscr{C}_{i}, \|\right), i=1,2,3$ be three 2-dimensional Laguerre planes. Suppose that $\mathscr{C}_{1}^{1}=\mathscr{C}_{2}^{1}=\mathscr{C}_{3}^{1}$ for some common circle $c_{0}$. Then $L_{1}\left|L_{2}\right| L_{3}=\left(\mathbb{S}^{1} \times \mathbb{R}, \mathscr{C}_{1}^{1} \cup \mathscr{C}_{1}^{2} \cup \mathscr{C}_{2}^{+} \cup \mathscr{C}_{3}^{-}, \mid\right)$is a 2-dimensional Laguerre plane (Figure 7).

We give a characterization of the fixed circle set of a pre-inversion. Let $L=$ $\left(\mathbb{S}^{1} \times \mathbb{R}, \mathscr{C}, \|\right)$ be a 2-dimensional Laguerre plane and let $\gamma$ be a pre-inversion of $L$. Let $\mathbb{S}^{2}$ be the 'two-point compactification' of the cylinder into the 2 -sphere $\mathbb{S}^{2}$ by two points $p_{+\infty}$ and $p_{-\infty}$. Let $D_{\gamma, L}$ be the incidence structure whose point set is $\mathbb{S}^{2}$ and whose circle set consists of all circles in $\mathscr{C}_{\gamma}$ and the sets that we arrive at by joining parallel classes with their image under $\gamma$ and the two points $p_{+\infty}$ and $p_{-\infty}$. We extend $\gamma$ to an involutory homeomorphism $\bar{\gamma}$ of $\mathbb{\$}^{2}$ to itself by setting $\bar{\gamma}\left(p_{+\infty}\right)=p_{-\infty}$. Now it is clear that all circles in $D_{\gamma, L}$ are homeomorphic to $\mathbb{S}^{1}$ and stay fixed under $\bar{\gamma}$. The quotient space $\mathbb{S}^{2} / \bar{\gamma}$, that is, the space we obtain by identifying the points of $\mathbb{S}^{2}$ via 


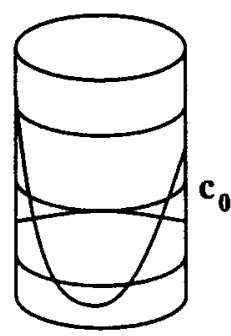

$L_{1}$

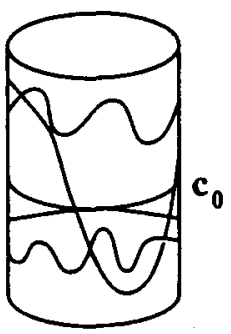

$L_{2}$

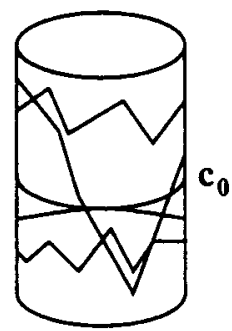

$L_{3}$

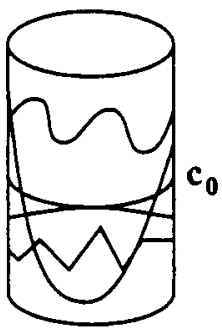

$L_{1}\left|L_{2}\right| L_{3}$

FIGURE 7

$\bar{\gamma}$, is the the real projective plane $P_{2}(\mathbb{R})$ (viewed as a topological space only). Let $D_{\gamma, L} / \bar{\gamma}$ denote the induced incidence structure on $P_{2}(\mathbb{R})$ from $D_{\gamma, L}$.

PROPOSITION 5. Let $L=\left(\mathbb{S}^{1} \times \mathbb{R}, \mathscr{C}, \|\right)$ a 2-dimensional Laguerre plane and let $\gamma$ be a pre-inversion of $L$. Then $D_{\gamma, L} / \bar{\gamma}$ is a 2-dimensional projective plane.

PROOF. From what we just said it is clear that $D_{\gamma, L} / \bar{\gamma}$ is an incidence geometry with the real projective plane as point set and sets homeomorphic to $\mathbb{S}^{1}$ as circles, or better, lines. By [Sa, Theorem 2.5] it now suffices to show that given two distinct points in this geometry there is a uniquely determined line connecting both points. This translates back to showing that the pencil of circles through an arbitrary point $p \in \mathbb{S}^{2}$ and $\bar{\gamma}(p)$ is a foliation of $\mathbb{S}^{2}$, that is, every point in $\mathbb{S}^{2} \backslash\{p, \bar{\gamma}(p)\}$ is contained in exactly one such circle. This is clearly the case.

For pre-reflections the corresponding result is not so nice and we cannot really say more than we already said in Lemma 4.

REMARKS 1. (1) The separating sets discussed in this section can be viewed as line sets of incidence geometries having various point sets. All these incidence geometries are closely related to 2 -dimensional projective planes:

Prop 1: The separating set is, essentially, the set of non-vertical lines of the derived affine plane at the point $p$ (as in Proposition 1). Remember that the point set of this affine plane is the cylinder from which the parallel class $p$ is contained in has been removed.

Prop 2*: The separating set is, essentially, the line set of a 'double cover' of a 2dimensional projective plane (cf. Proposition 5, above). The point set of this incidence geometry is the cylinder. 
Prop 3*: The separating set is, essentially, the set of non-vertical lines in a 'double cover' of a strip in a 2-dimensional affine plane. The point set of this incidence geometry is again the cylinder.

Prop 4: The separating set minus the circle $c_{0}$ splits into two parts $O$ and $U$ where $O$ and $U$ are the sets of circles that touch $c_{0}$ from above and below, respectively. Both $O$ and $U$ are block sets of so-called cylinder semi-biplanes (cf. [Po]). The point set of the first (second) cylinder semi-biplane is the set of points on the cylinder that are situated above (below) $c_{0}$. Given any cylinder semi-biplane there exists a 2-dimensional projective plane that is the 'double cover' of the cylinder semi-biplane (cf. [Po]).

(2) Again, the circle set of a given 2-dimensional Laguerre plane is homeomorphic to $\mathbb{R}^{3}$ : Here is an easy way to visualize this fact (cf. [Gr2, 3.8 (5b)]): Let $\Pi_{i}$, $i=1,2,3$ be three distinct parallel classes every one of which having been identified in a natural way with $\mathbb{R}$. Then a circle $c$ gets identified with the point $\left(x_{1}, x_{2}, x_{3}\right) \in \mathbb{R}^{3}$ where $x_{i}$ is the point of intersection of $c$ with $\Pi_{i}$.

Now it is easy to see that the separating sets discussed in Proposition $1,2^{*}$, and $3^{*}$ are closed subsets of the circle set homeomorphic to $\mathbb{R}^{2}$. In the case of Proposition 1, let $\Pi_{3}$ be the parallel class the point $p$ is contained in and let $\Pi_{1}$ and $\Pi_{2}$ be chosen arbitrarily. In the case of Propositions $2^{*}$ and $3^{*}$, let $\Pi_{1}$ and $\Pi_{2}$ be two parallel classes that are not fixed by $\gamma$ and such that $\Pi_{1} \neq \gamma\left(\Pi_{2}\right)$. We know, by axiom L1 in the first case and Lemma 4(1) in the last two cases, that, given one point each in $\Pi_{1}$ and $\Pi_{2}$, there is exactly one line in $\mathscr{C}_{\gamma}$ that contains both of them. Since the sets $\mathscr{C}_{\gamma}$, viewed as incidence geometries, are topological, this implies that $\mathscr{C}_{p}$ and $\mathscr{C}_{\gamma}$, as subsets of $\mathbb{R}^{3}$, are the graphs of continuous functions $\mathbb{R}^{2} \rightarrow \mathbb{R}$, that is, graphs of a functions from the $x_{1} x_{2}$-plane to the $x_{3}$-axis of $\mathbb{R}^{3}$. Hence they have the required properties.

We remark that the separating set in Proposition 4 is homeomorphic to a double cone (the vertex is the circle $c_{0}$ that gets touched). The proof of this fact relies on results in [Po].

All separating sets separate the circle set into open connected components: two in the first three cases, three in the last.

(3) Let $L_{1}$ and $L_{2}$ be ovoidal Laguerre planes. Let $\gamma_{1}$ and $\gamma_{2}$ be two pre-inversions of $L_{1}$ and $L_{2}$, respectively, that come from inner points of the respective cylinders in the 3-dimensional model, as described in Section 2. Then $D_{\gamma_{1}, L_{1}} / \bar{\gamma}_{1}$ and $D_{\gamma_{2}, L_{2}} / \bar{\gamma}_{2}$ are both isomorphic to the classical 2-dimensional projective plane, that is, the Desarguesian projective plane over the reals. Now it is not difficult to construct a homeomorphism $h$ from the first cylinder onto the second that maps verticals to verticals and such that $h \circ \gamma_{1} \circ h^{-1}=\gamma_{2}$. Therefore the two 2-dimensional Laguerre planes $h\left(L_{1}\right)$ and $L_{2}$ share the the same pre-inversion and Proposition $2^{*}$ can be applied to construct a new 2-dimensional Laguerre plane.

A similar statement can be made if $\gamma_{1}$ and $\gamma_{2}$ are two pre-reflections of $L_{1}$ and 
$L_{2}$, respectively, that come from exterior points of the respective cylinders in the 3-dimensional model.

Vertical lines. We continue the discussion from the beginning of this section. Every vertical line in $\mathbb{R}^{3}$ gives rise to a special kind of partition of the circle set. The elements of the partition are the sets of circles that correspond to the points in the line. So, every parallel class on the cylinder gives rise to a partition of the circle set into affine planes.

Every vertical line inside the cylinder gives rise to a partition into 'double covers' of 2-dimensional projective planes, and so on.

Similar partitions of the Mäurer planes arise in the same way.

\section{Vertical planes - Resolutions of 2-dimensional Laguerre planes}

Non-vertical lines. In the classical Laguerre plane, or more generally, in ovoidal planes, a non-vertical secant line of the cylinder in $\mathbb{R}^{3}$ corresponds to the pencil of circles through its two points of intersection with the cylinder. A non-vertical tangent line corresponds to a pencil of tangent circles in its point of tangency with the cylinder. An exterior line corresponds to a flock, that is, to a set of circles such that every single point on the cylinder is contained in exactly one circle in the set.

If we have a look at the Moulton plane that is used in the Mäurer construction and suppose that this Moulton plane does not intersect the cylinder, then any bent line in this Moulton plane gives rise to a flock in the corresponding Mäurer plane. This flock is just the set of all intersections of the bent planes that contain this bent line with the cylinder.

Resolutions. Every vertical plane in $\mathbb{R}^{3}$ gives rise to a special kind of partition of the circle set. The elements of the partition are the sets of circles that correspond to the lines in the plane. If the plane intersects the cylinder, it is clear what these partitions look like, and it is also clear what their counterparts in the non-classical Laguerre planes are.

The most interesting such partitions arise from planes that do not intersect the cylinder. These are partitions of the circle set into flocks. Such a partition is called a resolution (cf. [Bo]).

The Moulton plane in the Mäurer construction gives rise to all three kinds of partitions depending how it is situated with respect to the cylinder.

Rosehr [Ro] proved that every 2-dimensional Laguerre plane contains a flock of circles, but it is still not clear whether for any 2-dimensional Laguerre plane there exists a resolution of its circle set into flocks.

We proceed by giving examples of resolutions of circle sets of some well-known 
examples of 2-dimensional Laguerre planes.

Let $L=\left(\mathbb{S}^{1} \times \mathbb{R}, \mathscr{C}, \|\right)$ be a 2 -dimensional Laguerre plane such that the group of all translations of the cylinder in the vertical direction is a subgroup of the group of automorphisms of this Laguerre plane. The ovoidal Laguerre planes are examples of such planes. Let $R$ be the set of all orbits of circles of this group. Then $R$ is clearly a resolution of the circle set of $L$.

Let $L=\left(\mathbb{S}^{1} \times \mathbb{R}, \mathscr{C}, \|\right)$ be a 2-dimensional Laguerre plane. We identify $\mathbb{S}^{1}$ with $\mathbb{R} \cup\{\infty\}$ in a natural way. Then all circles in $L$ are graphs of continuous functions $\mathbb{R} \rightarrow \mathbb{R}$ to which some point on the infinite parallel class $\Pi_{\infty}=\{\infty\} \times \mathbb{R}$ has been adjoined. Using a stereographic projection as the identifying map, the circles in a Mäurer plane can be arranged to arise as follows (cf. [LP, Ha]):

$$
\begin{aligned}
& \left\{(x, y) \in \mathbb{R}^{2} \mid y=a f(x)+b x+c, x<0\right\} \\
& \quad \cup\left\{(x, y) \in \mathbb{R}^{2} \mid y=h(a) f(x)+b x+c, x \geq 0\right\} \cup\{(\infty, a)\}, a, b, c \in \mathbb{R}
\end{aligned}
$$

where $h: \mathbb{R} \rightarrow \mathbb{B}$ is a fixed strictly increasing homeomorphism and $f$ is a differentiable function such that $f^{\prime}$ is a strictly increasing homeomorphism and such that $f(0)=f^{\prime}(0)=0$. We note that if $h$ is the identity, then we are dealing with an ovoidal plane and if in addition $f(x)=x^{2}$, then we are dealing with the classical Laguerre plane. All these planes are examples of Laguerre planes such that all translations of $\mathbb{R}^{2}$ in the vertical direction

$$
v_{c}: \mathbb{R}^{2} \cup(\{\infty\} \times \mathbb{R}) \rightarrow \mathbb{R}^{2} \cup(\{\infty\} \times \mathbb{R}):(x, y) \mapsto\left\{\begin{array}{ll}
(x, y) & \text { for } x=\infty \\
(x, y+c) & \text { for } x \in \mathbb{R}
\end{array},\right.
$$

$c \in \mathbb{R}$, are automorphisms. Further examples of such planes can be found in [AG, Ha, $\mathrm{LP}, \mathrm{Kl}, \mathrm{Po}, \mathrm{St} 3$ ]. Let $T$ be the set of all tangent pencils of circles at the origin of $\mathbb{R}^{2}$ and let $\left\{h_{t}\right\}_{t \in T}$ be a set of strictly increasing homeomorphisms of $\mathbb{R}$. Let $f_{t, a}: \mathbb{R} \rightarrow \mathbb{R}$, $t \in T, a \in \mathbb{R}$ be the function that describes the uniquely determined circle in $t$ through the point $(\infty, a)$, and let $r_{t, c}, t \in T, c \in \mathbb{R}$ be the set that consists of the following circles:

$$
\left\{(x, y) \in \mathbb{R} \mid y=f_{t, a}(x)+h_{t}(a)+c\right\} \cup\{(\infty, a)\}, \quad a \in \mathbb{R} .
$$

Clearly, every such set of circles is a flock and $R=\left\{r_{t, c} \mid t \in T, c \in \mathbb{R}\right\}$ is a resolution of our Laguerre plane. For example, let us look at the classical Laguerre plane and let all homeomorphisms be equal to the identity homeomorphism. Then we can identify the abstract set $T$ with $\mathbb{R}$ such that $r_{t, c}$ consists of the circles $\left\{(x, y) \in \mathbb{R}^{2} \mid y=\right.$ $\left.a x^{2}+t x+a+c\right\} \cup\{(\infty, a)\}, a \in \mathbb{R}$.

It is also possible to construct resolutions of the examples of 2-dimensional Laguerre planes in [St2] and [St3] that have trivial automorphism groups.

We note that in any affine plane any parallel class is a 'flock' of lines, and that the set of all parallel classes forms a 'resolution' of the affine plane. 


\section{Separating sets in quadrangles}

This section is a continuation of our discussion of separating sets from Section 3 .

Introduction to quadrangles and their relationship with Laguerre planes. In 1981 Forst [Fo] proved that the so-called Lie-geometry of a 2-dimensional Laguerre plane is a 3-dimensional quadrangle, that is, a special kind of (topological) generalized quadrangle.

We now give a brief summary of this relationship following the exposition in [Sch1].

A generalized quadrangle is an incidence structure $Q=(P, \mathscr{L}, I)$ consisting of a point set $P$, a line set $\mathscr{L}$ and an incidence relation $I \subseteq P \times \mathscr{L}$ satisfying the following axioms:

(Q1) Any two distinct points are incident with at most one line.

(Q2) For every anti-flag $(p, L) \in P \times \mathscr{L} \backslash I$ there exists exactly one flag $(q, M) \in I$ such that $(p, M) \in I$ and $(q, L) \in I$.

(Q3) (Thickness) Every point is incident with at least three lines, and dually, every line is incident with at least three points.

Axiom Q2 yields two mappings $\pi: P \times \mathscr{L} \backslash I \rightarrow P$ and $\lambda: P \times \mathscr{L} \backslash I \rightarrow \mathscr{L}$ with $\pi(p, L)=q$ and $\lambda(p, L)=M$. The definition is self-dual so the geometric structure with points and lines interchanged is again a generalized quadrangle, called the dual quadrangle.

Usually we will consider a line in a quadrangle to be a subset of the point set $P$. All the information about the incidence in the quadrangle is then contained in the pair $(P, \mathscr{L})$ and we will refer to the quadrangle in this way.

Two points are said to be collinear if they can be joined by a line. The line connecting two collinear points $p, q$ is denoted by $p \vee q$. The set of points collinear with a given point $p$ is denoted by $p^{\perp}$.

A 3-dimensional quadrangle is a generalized quadrangle where $P$ and $\mathscr{L}$ carry compact Hausdorff topologies such that the maps $\pi$ and $\lambda$ become continuous and such that $P$ is 3 -dimensional. In a 3 -dimensional quadrangle lines, considered as point sets, and line pencils are homeomorphic to 1-spheres. Of course, the dual of a 3-dimensional quadrangle is also 3-dimensional.

The 3-dimensional projective space $P_{3}(\mathbb{R})$ over $\mathbb{R}$ carries a natural topology such that it becomes a topological projective space with compact point and line spaces. Let $\beta$ be a symplectic form on this space. The incidence geometry $Q(\beta)$ whose point set is the point set of $P_{3}(\mathbb{R})$ and whose lines are the totally isotropic lines is a generalized quadrangle. Moreover, the dual of this quadrangle is antiregular, that is, for every choice of three mutually non-collinear points there are either no or exactly two points collinear to all three points. The topology on $P_{3}(\mathbb{R})$ induces a natural topology on 
the symplectic quadrangle $Q(\beta)$. With the induced topologies the point space as well as the line space become compact and $Q(\beta)$ and its dual turn into 3-dimensional quadrangles.

The construction of a 3-dimensional quadrangle from a 2-dimensional Laguerre plane $L=\left(\mathbb{S}^{1} \times \mathbb{R}, \mathscr{C}, \|\right)$ is as follows: The points of the quadrangle are the points of the Laguerre plane, the circles of the Laguerre plane and a symbol $\infty$. An extended parallel class is a parallel class to which the point $\infty$ is added and an extended tangent circle pencil is a set of circles touching in a common point together with this point. The lines of the quadrangle are the extended parallel classes and the extended tangent circle pencils. The incidence is the natural one. The topologies on $P$ and $\mathscr{L}$ of the quadrangle are inherited from the topologies of the Laguerre plane in a rather intricate way such that the collection of all points of the quadrangle that stem from circles of the Laguerre plane is open in $P$ and such that the topology induced from $P$ on this set of points is the given topology of $\mathscr{C}$ (remember that, by Remark $1.2, \mathscr{C}$ is homeomorphic to $\mathbb{R}^{3}$ ). The same construction works for finite Laguerre planes of odd order (see [PT1, Ta]), and for 4-dimensional Laguerre planes (cf. [Sch3, Chapter 3]).

Conversely, let $Q=(P, \mathscr{L})$ be a generalized quadrangle and let $p \in P$. The derivation $Q_{p}$ of $Q$ at $p$ is the incidence structure $\left(p^{\perp},\left\{p^{\perp} \cap q^{\perp} \mid q \in P \backslash p^{\perp}\right\}\right)$. If $p$ is taken to be $\infty$ this construction reverses the above one. In general, $Q_{p}$ is anything but a Laguerre plane. However, for antiregular quadrangles one always obtains Laguerre planes. Also, in general, if $Q$ is a 3-dimensional quadrangle, then it is antiregular if and only if its dual is not antiregular. If a 3-dimensional quadrangle is antiregular, then the derivation at any point $p$ is a 2-dimensional Laguerre plane and $p^{\perp}$ is homeomorphic to the one-point compactification of the cylinder. If it is not, then $p^{\perp}$ is homeomorphic to the real projective plane.

Separating sets in 3-dimensional antiregular quadrangles. In Section 3 we considered certain separating sets of the circle sets of 2-dimensional Laguerre planes. We now investigate what these separating sets correspond to in the associated 3dimensional quadrangles.

We begin with the separating set described in Proposition 1. Figure 8 depicts the point set of the quadrangle $Q=(P, \mathscr{L})$ that is associated with the Laguerre plane $L=\left(\mathbb{S}^{1} \times \mathbb{R}, \mathscr{C}, \|\right)$.

The point set of $Q$ consists of the circle set of $L$, which, by Remark 1.2 , is a topological space homeomorphic to $\mathbb{R}^{3}$ (in the picture this is the cube), and the onepoint compactification of the point set of $L$ by the point $\infty$, that is, a 2-dimensional topological space (in the picture this is the quadrilateral below the cube). These two sets are glued together in a (topologically sound) way that is of no importance for our discussion. The separating set corresponds (same remark) to a subset of the $\mathbb{R}^{3}$ homeomorphic to $\mathbb{R}^{2}$ that separates the $\mathbb{R}^{3}$. into two open components. The picture 
also shows how lines in the quadrangle that correspond to different parallel classes and tangent circle bundles are situated within the quadrangle.

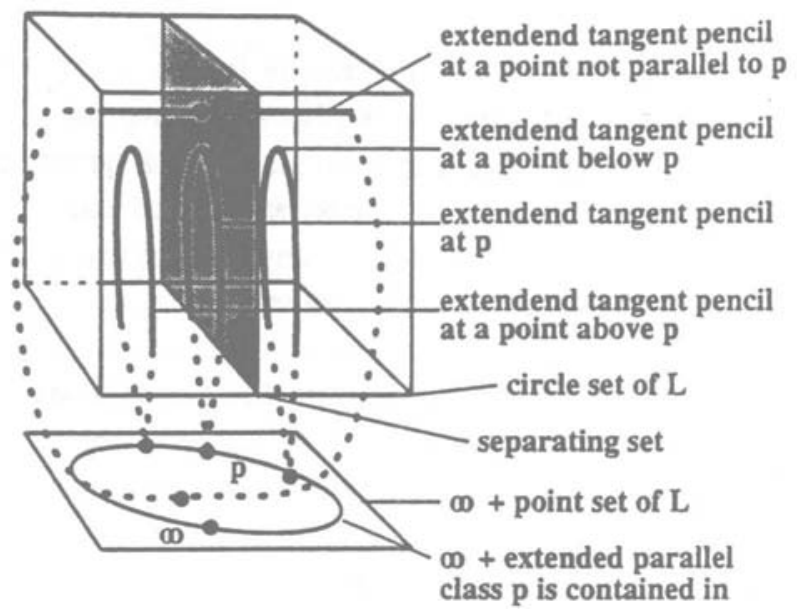

FIGURE 8

We now describe the objects we are interested in in the language of quadrangles.

A separating set in the quadrangle $Q$ that corresponds to the separating set $\mathscr{C}_{p}$ in the Laguerre plane $L$ is $\infty^{\perp} \cup p^{\perp}$ : Notice that the line in $Q$ that corresponds to a tangent circle pencil in $p$ is completely contained in the separating set $\mathscr{C}_{p}$ (except, of course, for the point $p$ itself). Clearly, $\mathscr{C}_{p}=p^{\perp} \backslash\{p \vee \infty\}$ and the points of the Laguerre plane correspond to $\infty^{\perp} \backslash\{\infty\}$. The set $p^{\perp} \backslash\{p \vee \infty\}$ is not a separating set of the point set of the quadrangle. Recall that when gluing together two Laguerre planes as in Proposition 1 both planes have the same point set and the same set of circles $\mathscr{C}_{p}$. Thus the separating set in the quadrangle that we are looking for is indeed $\infty^{\perp} \cup p^{\perp}$.

The connected components $P_{1}$ and $P_{2}$ of $P \backslash\left(\infty^{\perp} \cup p^{\perp}\right)$ : The parallel class of $p$ plus the point $\infty$ form a line in $Q$. This line $p \vee \infty$ is homeomorphic to $\mathbb{S}^{1}$. Hence $(p \vee \infty) \backslash\{p, \infty\}$ has two connected components $C_{1}$ and $C_{2}$. All points not belonging to $\infty^{\perp} \cup p^{\perp}$ (our new separating set) come from circles of the Laguerre plane. Each such circle intersects the parallel class of $p$ either above or below $p$. In the quadrangle this means that each point of $P \backslash\left(\infty^{\perp} \cup p^{\perp}\right)$ is collinear to a point of $(p \vee \infty) \backslash\{\infty, p\}$. It follows that the connected components $P_{1}$ and $P_{2}$ of $P \backslash\left(\infty^{\perp} \cup p^{\perp}\right)$ are precisely the collection of all points that are collinear to a point of $C_{1}$ or $C_{2}$, respectively.

The partition of the line set of the quadrangle with respect to the new separating set: With respect to the new separating set we distinguish four essentially different kinds of lines in the quadrangle.

(1) Lines that are completely contained in the separating set. These are the lines that arise from parallel classes and the lines that arise from tangent circle pencils at the point $p$. 
(2) Lines that (except for one point) are completely contained in $P_{1}$. Every such line arises from a tangent circle pencil at a point above $p$.

(3) Lines that (except for one point) are completely contained in $P_{2}$. Every such line arises from a tangent circle pencil at a point below $p$.

(4) Lines that have points in common both with $P_{1}$ and $P_{2}$. Every such line arises from a tangent circle pencil at a point $q$ not parallel to $p$ and has precisely two points in common with the separating set ( $q$ and the unique circle through $p$ ).

Let $\mathscr{L}^{0}, \mathscr{L}^{11}, \mathscr{L}^{12}$ and $\mathscr{L}^{2}$ denote the set of lines of the first, second, third and fourth kind, respectively. Clearly, $\mathscr{L}^{0} \cup \mathscr{L}^{11} \cup \mathscr{L}^{12} \cup \mathscr{L}^{2}$ is a partition of the line set of $Q$.

Let $Q^{*}=\left(P, \mathscr{L}^{*}\right)$ be the quadrangle that corresponds to $L^{*}$ (in Proposition 1) and let $Q \mid Q^{*}$ be the quadrangle that corresponds to $L \mid L^{*}$. If $l \in \mathscr{L}^{2}$, let $l^{*}$ be the unique line in $\mathscr{L}^{* 2}$ that intersects $p^{\perp} \cup \infty^{\perp}$ in the same two points as $l$, let $l \mid l^{*}$ be the set that coincides with $l$ on $P_{1} \cup p^{\perp} \cup \infty^{\perp}$ and with $l^{*}$ on $P_{2}$. Finally, let $\mathscr{L}^{2} \mid \mathscr{L}^{* 2}$ be the set of all such sets.

The gluing together of $L$ and $L^{*}$ along the set of circles $\mathscr{C}_{p}$ corresponds to the gluing together of two chunks of points, taken from $Q$ and $Q^{*}$, along a common subset of their point sets, that is, on each of the two connected components of the complement of the (new) separating set the geometry of $Q \mid Q^{*}$ is induced from one of the two quadrangles and lines of different components are glued together at their intersection with the separating set. The line set of $Q \mid Q^{*}$ is $\mathscr{L}^{0} \cup \mathscr{L}^{11} \cup \mathscr{L}^{* 12} \cup\left(\mathscr{L}^{2} \mid \mathscr{L}^{* 2}\right)$.

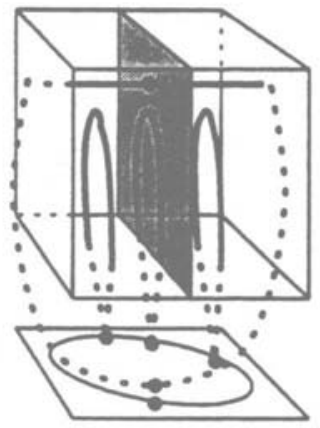

$Q$

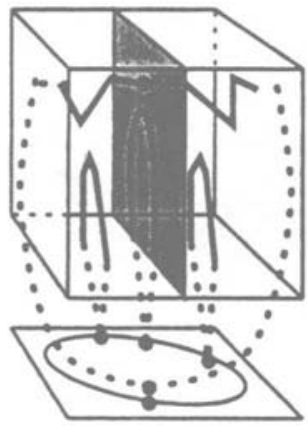

$Q^{*}$

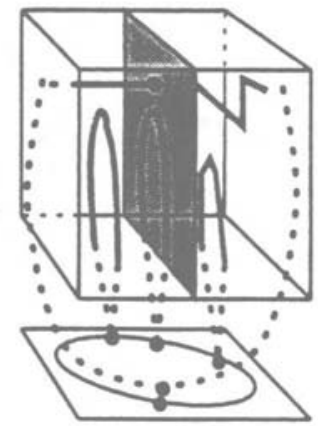

$Q \mid Q^{*}$

FIGURE 9

REMARK 2. It is not known whether the point sets of all 3-dimensional antiregular quadrangles are homeomorphic. We only know that such a point set is a compact 3-dimensional space that is the disjoint union of two sets; one homeomorphic to $\mathbb{R}^{3}$, the other one homeomorphic to the one-point compactification of the cylinder. So, when we denote all the point sets of 3-dimensional quadrangles by $P$, we do not think 
of $P$ as a topological space, we rather think of it as a disjoint union of two sets like this without worrying about how exactly these two sets are glued together.

The interpretation of the separating sets constructed in Propositions $2^{*}$ and $3^{*}$ is essentially the same as in the case on Proposition 1 since the separating sets are still homeomorphic to $\mathbb{R}^{2}$. So again the new separating set in the quadrangle is $\mathscr{C}_{\gamma} \cup \infty^{\perp}$. Notice that, although $\mathscr{C}_{\gamma}$ cannot be described as easily in geometric terms as $\mathscr{C}_{p}$, every extended tangent circle pencil through a point $q$ that is not fixed by $\gamma$ contains one element in $\mathscr{C}_{\gamma}$. It also contains the point $q$. Hence the line in the associated quadrangle that corresponds to this tangent circle pencil intersects the (new) separating set $\infty^{\perp} \cup \mathscr{C}_{\gamma}$ in two points. A tangent circle pencil through a point that is fixed by $\gamma$ (this only happens if $\gamma$ is a pre-reflection) is completely contained in either $\mathscr{C}_{\gamma}, \mathscr{C}_{\gamma^{+}}$or $\mathscr{C}_{\gamma^{-}}$. Hence the picture for the case in which $\gamma$ is a pre-reflection is basically the same as in Figure 9 (just delete the distinguished line $p \vee \infty$ in Figure 9). In the case in which $\gamma$ is a pre-inversion we arrive at the picture as in Figure 10.

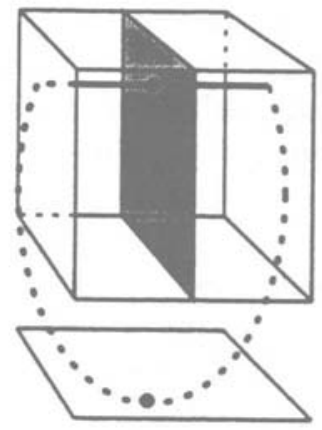

$Q$

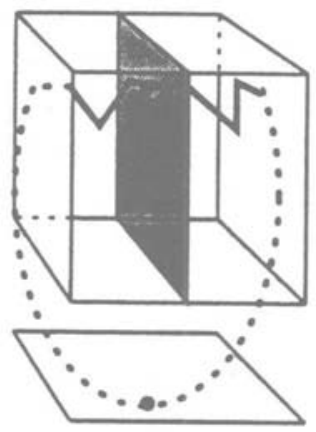

$Q^{*}$

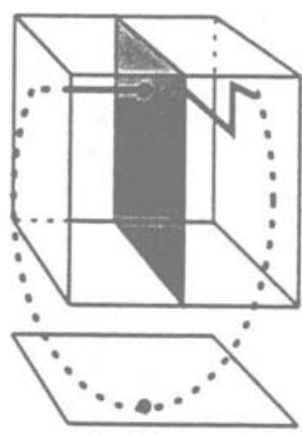

$Q \mid Q^{*}$

FIGURE 10

REMARK 3. In the situations described in Propositions 2 and 3 we can say a little more. An inversion $\gamma$ of the Laguerre plane $L$ can be extended to an involutory automorphism $\gamma^{\prime}$ of the associated quadrangle $Q$. The set of fixed points $F$ of $\gamma^{\prime}$ is $\mathscr{C}_{\gamma} \cup\{\infty\}$ which, as a topological space, is homeomorphic to the 2-sphere $\mathbb{S}^{2}$ (remember that $\mathscr{C}_{\gamma}$ is homeomorphic to $\mathbb{R}^{2}$ ). Furthermore, $F$ has the property that every line of $Q$ intersects $F$ in precisely one point, for example, each tangent circle pencil contains exactly on circle in $\mathscr{C}_{\gamma}$. It turns out that the incidence structure $\left(F,\left\{F \cap x^{\perp} \mid x \in P \backslash F\right\}\right)$ is a 2-dimensional Möbius plane and that every 2dimensional Möbius plane can be constructed in this way from a 3-dimensional quadrangle admitting such an involutory automorphism $\gamma^{\prime}$; cf. [Gr4] and [Sch3, Chapter 4]. 
Similarly, a reflection $\gamma$ can be extended to an involutory automorphism $\gamma^{\prime}$ of the associated quadrangle $Q$. The set of fixed points $F$ of $\gamma^{\prime}$ is $\mathscr{C}_{\gamma} \cup \Pi_{0} \cup \Pi_{\infty} \cup\{\infty\}$ which, as a topological space, is homeomorphic to the torus $\mathbb{S}^{1} \times \mathbb{S}^{1}$. The set $F$ is the point set of a 2-dimensional Minkowski plane whose parallel classes are the lines contained in $F$. Circles in one connected component of the circle set of the Minkowski plane are of the form $F \cap x^{\perp}, x \in P \backslash F$. Circles in the other component are defined in a more intricate way (cf. [Sch3, Chapter 5]).

Finally, we look at the situation described in Proposition 4. Let $Q_{i}, i=1,2,3$ be the quadrangle associated with $L_{i}$ and let $Q_{1}\left|Q_{2}\right| Q_{3}$ be the quadrangle associated with $L_{1}\left|L_{2}\right| L_{3}$. Consider the collection $\mathscr{C}^{1}$ of all circles that touch $c_{0}$. In $Q_{i}$ the circle $c_{0}$ is a point and the points on $c_{0}$ and all circle in $\mathscr{C}^{1}$ are precisely $c_{0}^{\perp}$. Hence as a separating set in the quadrangle we have $\infty^{\perp} \cup c_{0}^{\perp}$. Written in this way this separating set looks exactly like the separating set $\infty^{\perp} \cup p^{\perp}$ that we considered earlier. There is a difference, though. Whereas $\infty$ and $p$ are collinear, $\infty$ and $c_{0}$ are not.

A tangent circle pencil in $L_{i}$ through a point $q$ not on $c_{0}$ contains precisely one circle in $\mathscr{C}^{1}$. Hence the line in $Q_{i}$ that corresponds to this pencil intersects the (new) separating set two times. Every such line has points in only two of the three components of $\mathbb{R}^{3} \backslash \mathscr{C}^{1}$. None has points in both $\mathscr{C}_{i}^{+}$and $\mathscr{C}_{i}^{-}$. So, basically, we arrive at the following picture of a quadrangle $Q=(P, \mathscr{L})$ associated with a Laguerre plane $\mathscr{L}=\left(\mathbb{S}^{1} \times \mathbb{R}, \mathscr{C}, \|\right)$ that contains the circle $c_{0}$ (Figure 11).

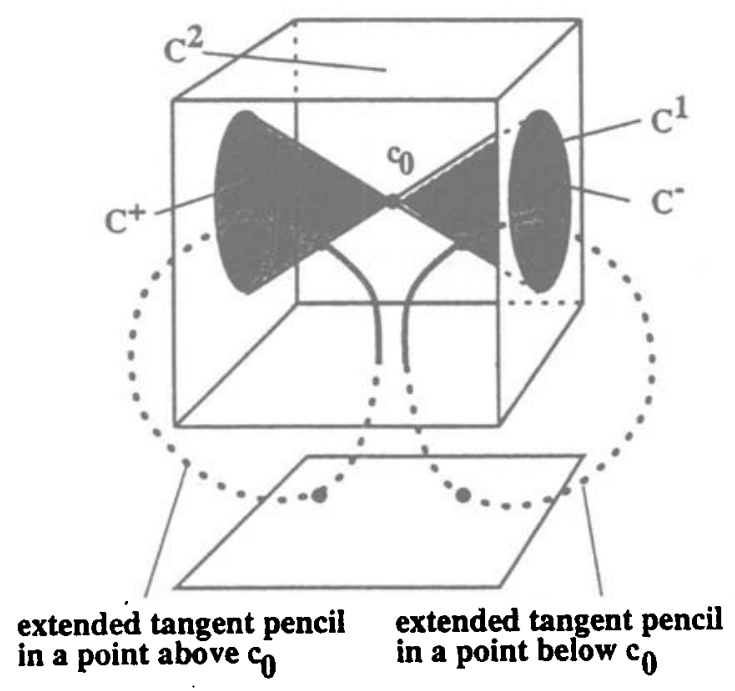

FIGURE 11

Finally, the parts of $Q_{1}, Q_{2}$ and $Q_{3}$ get glued together into $Q_{1}\left|Q_{2}\right| Q_{3}$ as follows. 


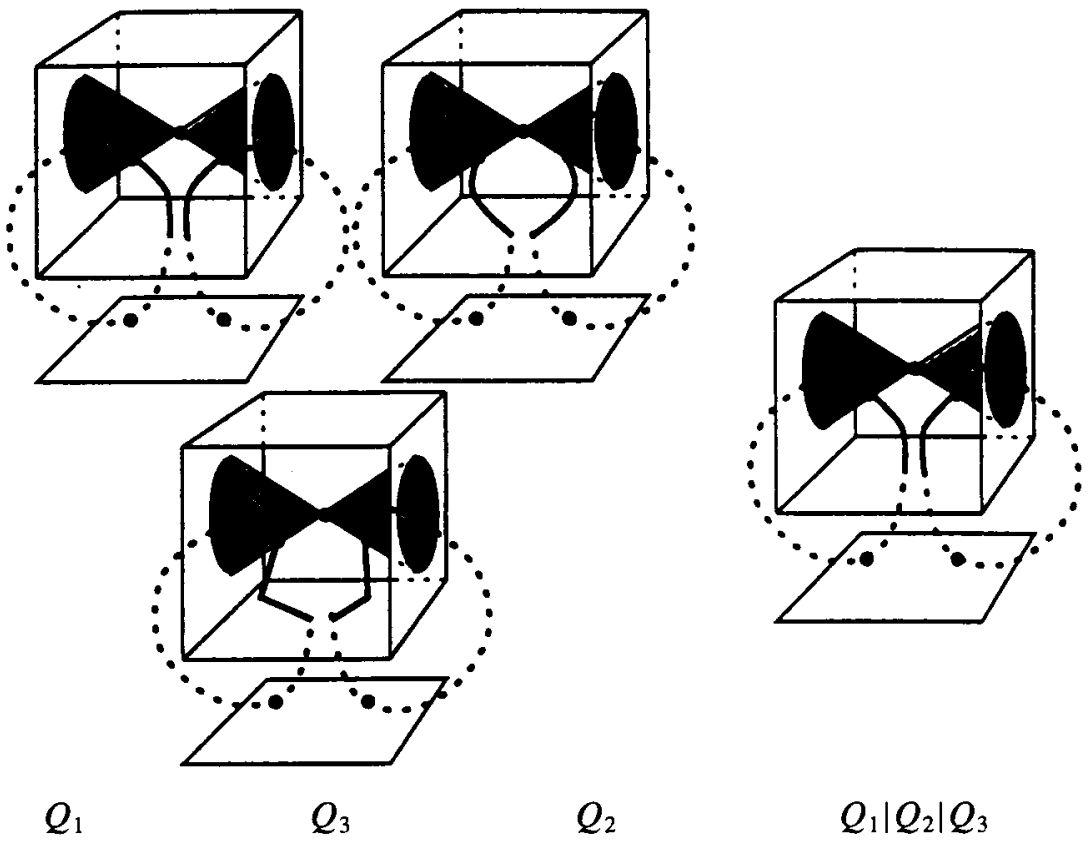

FIGURE 12

\section{References}

[AG] R. Artzy and H. Groh, 'Laguerre and Minkowski planes produced by dilatations', J. Geom. 26 (1986), 1-20.

[Bo] A. Bonisoli, 'On resolvable finite Minkowski planes', J. Geom. 36 (1989), 1-7.

[Fo] M. Forst, 'Topologische 4-Gone', Mitt. Math. Sem. Giessen 147 (1981), 65-129.

[Gr1] H. Groh, 'Topologische Laguerreebenen I', Abh. Math. Sem. Univ. Hamburg 32 (1968), 216231.

[Gr2] - ' 'Topologische Laguerreebenen II', Abh. Math. Sem. Univ. Hamburg 34 (1970), 11-21.

[Gr3] — ' 'Ovals and non-ovoidal Laguerre planes', J. Reine Angew. Math. 267 (1974), 50-66.

[Gr4] - 'Flat Moebius and Laguerre planes', Abh. Math. Sem. Univ. Hamburg 40 (1974), 64-76.

[Ha] E. Hartmann, 'Moulton Laguerre-Ebenen', Arch. Math. 27 (1976), 424-435.

[K1] R. Kleinewillinghöfer, Eine Klassifikation der Laguerre-Ebenen (Dissertation, Darmstadt, 1979).

[LP] R. Löwen and U. Pfüller, 'Two-dimensional Laguerre planes over convex functions', Geom. Dedicata 23 (1987), 73-85.

[Mäu] H. Mäurer, 'Eine Kennzeichnung halbovoidaler Laguerre-Geometrien', J. Reine Angew. Math. 253, 203-213.

[Mou] F. R. Moulton, 'A simple non-desarguesian plane geometry', Trans. Amer. Math. Soc. 3 (1902), 192-195.

[PT1] S. E. Payne and J. A. Thas, 'Generalized quadrangles with symmetry', Simon Stevin 49 (1970), 3-32 and 81-103.

[PT2] - Finite Generalized Quadrangles, Research Notes in Math. 110 (Pitman, Boston, 1984).

[Po] B. Polster, 'Semi-biplanes on the cylinder', Geom. Dedicata 58 (1995), 145-160. 
[PRS] B. Polster, N. Rosehr and G. F. Steinke, 'Halfovoidal Laguerre planes', J. Geom., to appear.

[PS1] B. Polster and G. F. Steinke, 'Criteria for two-dimensional circle planes', Beiträge Algebra Geom. 35 (1994), 181-191.

[PS2] _ _ 'Cut and paste in 2-dimensional circle planes', Can. Math. Bull 38 (1995), 469-480.

[Ro] N. Rosehr, 'Flocks in 2-dimensional circle planes', (preprint).

[Sa] H. Salzmann, 'Topological planes', Adv. Math. 2 (1967), 1-60.

[Sch1] A. E. Schroth, 'Three-dimensional quadrangles and flat Laguerre planes', J. Geom. 36 (1990), 365-373.

[Sch2] _ _ 'The Appolonius Problem in flat Laguerre planes', J. Geom. 42 (1991), 141-147.

[Sch3] Topological circle planes and topological quadrangles (Habilitationsschrift, Technische Universität Braunschweig, 1994).

[Sch4] _ - 'The Appolonius problem in four-dimensional Laguerre planes', J. Geom. 51 (1994), 138-149.

[St1] G. F. Steinke, 'Topological affine planes composed of two Desarguesian halfplanes and projective planes with trivial collineation group', Arch. Math. 44 (1985), 472-480.

[St2] _ _ 'Semiclassical topological flat Laguerre planes obtained by pasting along a circle', Resultate Math. 12 (1987), 207-221.

[St3] _ _ 'Semiclassical topological flat Laguerre planes obtained by pasting along two parallel classes', J. Geom. 32 (1988), 133-156.

[Th] J. A. Thas, 'Circle Geometries and generalized quadrangles', in: Finite geometries (Winnipeg, Man., 1984), Lecture Notes Pure Appl. Math. 103 (Dekker, New York, 1985) pp. 327-352.

[Val] G. Valette, 'Structures d'ovale topologique sur le cercle', Bull. Acad. R. Belg. 51 (1965), 586-597.

Department of Mathematics and Statistics

University of Canterbury

Christchurch

New Zealand

e-mail: bmp and gfs@math.canterbury.ac.nz 